\title{
All-or-none (or something in between) transition of DNA
}

Article

Accepted Version

Hayashi, Y. (2004) All-or-none (or something in between) transition of DNA. Europhysics Letters, 68 (4). pp. 536-542. ISSN 0295-5075 doi: https://doi.org/10.1209/epl//2004-10246-x Available at https://centaur.reading.ac.uk/30821/

It is advisable to refer to the publisher's version if you intend to cite from the work. See Guidance on citing.

To link to this article DOI: http://dx.doi.org/10.1209/epl/i2004-10246-x

Publisher: Institute of Physics Publishing

All outputs in CentAUR are protected by Intellectual Property Rights law, including copyright law. Copyright and IPR is retained by the creators or other copyright holders. Terms and conditions for use of this material are defined in the End User Agreement.

\section{www.reading.ac.uk/centaur}

\section{CentAUR}

Central Archive at the University of Reading

Reading's research outputs online 


\title{
All-or-none (or something in between) transition of DNA
}

\author{
YOSHIKATSU HAYASHI ${ }^{1}$ \\ 1 Physical Chemistry 1, Center for Chemistry and Chemical Engineering, Lund Uni- \\ versity, P.O. Box 124, S-22100 Lund, Sweden
}

PACS. 61.25.Hq - Macromolecular and polymer solutions; polymer melts; swelling.

\begin{abstract}
DNA compaction can be caused by multivalent ions as condensing agents. Both discontinuous (all-or-none) and continuous (pearl-necklace structure) transitions have been observed in experiments as the concentration of the condensing agent was increased. We have investigated the DNA transition by analytical calculations in the infinite-chain limit. A mechanism for pearl-necklace structures could be a combinatorial entropy term, which favours a mixture of globules and coils in a single chain. However, when a surface term is taken into account, it gives rise to a discontinuous transition. We also consider the role of surface charges on the globule.
\end{abstract}

Introduction. - In the cell, genomic DNA generally exists in a compact state. DNA compaction can be caused by a variety of condensing agents. This compaction of a semi-flexible polyelectrolyte has attracted much interest from physicists and chemists as an experimental system for the transition between coil and globule states [1-3]. Single DNA molecules can be visualised by fluorescence microscopy. Early reports showed that DNA compaction is an allor-none transition and was described as a first order transition [4-6]. However, recent studies have revealed a more detailed scenario $[7,8]$. DNA compaction is initiated by the formation of several nucleation centers along DNA and a partial globule state exists as a stable state; i.e., coil and globule states can coexist in a single chain. Depending on the concentration of the condensing agent, the most stable state changes from a coil state to partial globule, to a fully collapsed state. The formation of similar pearl-necklace structures has also been observed experimentally for other polyelectrolytes [9] and in simulation studies [10,11]. A repulsive electrostatic self-energy of the globule competes with the surface effect of a cohesive energy. This is called Rayleigh instability. The question arises whether the coil-globule transition is an all-or-none transition or a continuous transition (something in between) with partial globules. In this paper, we focus on the long-chain limit to get insight into the basic nature of the coil-globule transition, separating out the finite-size effect. We discuss what gives 'all or none' and what gives 'in between'.

Theory. - We adopt a phenomenological model for the DNA transition with (i) two conformational states to represent a coil part and a globule part and (ii) two states for the condensing agent [12], bound to the chain or free in the bulk. In experiments, there is always monovalent salt, which competes with the condensing agent. Here we consider monovalent salt only as a background screening. 'Counterion' simply means condensing agent. To include the

(c) EDP Sciences 


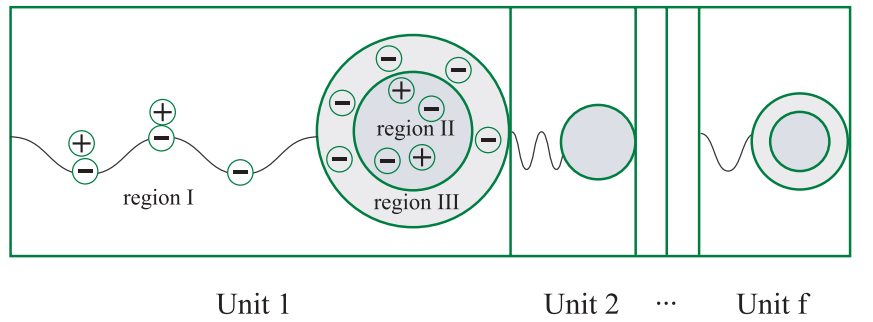

Fig. 1 - Schematic picture of the system.

degrees of freedom that allow for pearl-necklace structures, i.e., several intra-chain globules connected by coil regions, the polymer chain is divided into $f$ units where each unit consists of a coil part and a globule part. In each unit, we have three regions, namely, the coil region denoted region $I$, and two regions in the globule part, a neutral core, region $I I$, and a charged shell, region $I I I$. Furthermore, $n_{I}$ is the number of monomers in a subchain between two successive globule regions, $n_{I I}$ is the number of monomers in the neutral core region of a globule, and $n_{I I I}$ is the number of monomers in the shell part. For the condensing agent, $m_{I}$ and $m_{I I}$ describe the number of molecules bound to region $I$ and region $I I$, respectively. A schematic picture is given in Fig. 1.

The periodic units are numbered $1,2,3, \cdots, i, \cdots, f$ from one end of the chain. The total numbers of counterions and monomers are obtained by summing up the numbers in the different units,

$$
\begin{aligned}
M & =\sum_{i=1}^{f} m_{I, i}+\sum_{j=1}^{f} m_{I I, j} \\
N & =\sum_{i=1}^{f} n_{I, i}+\sum_{j=1}^{f} n_{I I, j}+\sum_{k=1}^{f} n_{I I I, k}
\end{aligned}
$$

where $M$ is the total number of counterions bound to the chain. The canonical partition function of the system with $N$ monomers is given by

$$
Z(M, f, N)=\sum_{m_{I}, m_{I I}, n_{I}, n_{I I}, n_{I I I}} \begin{aligned}
& p\left(m_{I, 1}, n_{I, 1}\right) q\left(m_{I I, 1}, n_{I I, 1}, n_{I I I, 1}\right) \cdots \\
& p\left(m_{I, f}, n_{I, f}\right) q\left(m_{I I, f}, n_{I I, f}, n_{I I I, f}\right)
\end{aligned}
$$

where $p\left(m_{I}, n_{I}\right)$ is the partition function of the coil part and $q\left(m_{I I}, n_{I I}, n_{I I I}\right)$ is the partition function of the globule part, assuming statistical independence of the two states. The difficult part here is that we have to count all the states for unit 1 , unit $2, \cdots$, unit $f$. In unit $i$, both the coil part (denoted $c_{i}$ ) and the globule part (denoted $g_{i}$ ) can vary in size. In the whole chain we have all the states, $c_{1}-g_{1}-\cdots c_{f}-g_{f}$. This creates a combinatorial entropy, $\Omega_{c-g}$, which is coupled to the energy of each possible state of $c_{1}-g_{1}-\cdots c_{f}-g_{f}$. To avoid this difficulty, we release the constraints for $M, f$, and $N$. First, we establish equilibrium with the condensing agent in a bulk solution by considering a grand canonical ensemble,

$$
\Xi(\lambda, f, N)=\sum_{M \geq 0} Z(M, f, M) \lambda^{M} .
$$

$\lambda$ is defined by $\lambda \equiv e^{\beta \mu}$, where $\mu$ is the chemical potential of the condensing agent, and $\beta \equiv 1 / k_{B} T$ with $k_{B}$ being Boltzmann's constant and $T$ is the absolute temperature. To 
further release the constraints, we write first and second generating functions by introducing conjugate variables; $\eta$ for the number of pearls, $f$, and $\xi$ for the number of monomers, $N$.

$$
\begin{aligned}
& \tilde{\Xi}(\lambda, \eta, N)=\sum_{f \geq 1} \sum_{M \geq 0} Z(M, f, N) \lambda^{M} \eta^{f}, \\
& \tilde{\tilde{\Xi}}(\lambda, \eta, \xi)=\sum_{N \geq 1} \sum_{f \geq 1} \sum_{M \geq 0} Z(M, f, N) \lambda^{M} \eta^{f} \xi^{N} .
\end{aligned}
$$

Substituting eqs. (1) to (3) into eq. (6) and calculating the infinite series with respect to the sum over $f$, we obtain the second generating function as,

$$
\tilde{\tilde{\Xi}}(\lambda, \eta, \xi)=\frac{P(\lambda, \xi) Q(\lambda, \xi) \eta}{1-P(\lambda, \xi) Q(\lambda, \xi) \eta}
$$

where

$$
\begin{aligned}
& P(\lambda, \xi)=\sum_{n_{I} \geq 0} \sum_{m_{I} \geq 0} p\left(m_{I}, n_{I}\right) \lambda^{m_{I}} \xi^{n_{I}} \\
& Q(\lambda, \xi)=\sum_{n_{I I} \geq 0} \sum_{n_{I I I} \geq 0} \sum_{m_{I I} \geq 0} q\left(m_{I I}, n_{I I}, n_{I I I}\right) \lambda^{m_{I I}} \xi^{n_{I I}} \xi^{n_{I I I}} .
\end{aligned}
$$

Since the number of monomers of a chain is always conserved, we have to go back to the first generating function by an inverse Laplace transformation,

$$
\tilde{\Xi}(\lambda, \eta, N)=\oint \frac{d \xi}{2 \pi i} \frac{P Q \eta}{1-P Q \eta} \xi^{-N} .
$$

From the residue theorem we get,

$$
\tilde{\Xi}(\lambda, \eta, N)=\xi_{0}(\lambda, \eta)^{-N} .
$$

The pole $\xi_{0}(\lambda, \eta)$ is a solution of

$$
1-P(\lambda, \xi) Q(\lambda, \xi) \eta=0 .
$$

The physical meaning of eq. (11) is $F_{\text {total }}=-k_{B} T \ln \tilde{\Xi}(\lambda, \eta, N)=N \mu_{\text {monomer }}(\lambda, \eta)$. The free energy of the system is equal to the 'total number of monomers times the chemical potential of a monomer'. This equation ensures the equilibrium condition for chain monomers in the coil and globule states. We also see that $\Omega_{c-g}$ and the contribution from the counterions are renormalised into the chemical potential of a monomer.

Now, we can calculate average properties for the DNA transition as a function of the chemical potential (concentration in the bulk) of the condensing agent. As an example, if we take the derivative of eq. (11) with respect to $\eta$, we can calculate the average number of pearls $f$ per monomer along the chain, $\langle f\rangle / N$, as

$$
\frac{\langle f\rangle}{N}=-\left.\frac{\partial \xi_{0}(\lambda, \eta)}{\partial \ln \eta}\right|_{\eta=1} .
$$

After taking the derivative with respect to $\eta, \eta$ is set equal to one (the chemical potential of a c-g unit is zero). This corresponds to turning off the field conjugate to $f$, since $\eta$ is introduced artificially to release the constraint on $f$. As another example, we will calculate the fraction 
of chain monomers in region II. If we look carefully at the exponent of $\xi$ in eq. (9), we note that $\xi^{n_{I I}}$ can be separated out as $\xi_{I I}^{n_{I I}}$. Therefore, $\left\langle n_{I I}\right\rangle / N$ is calculated by taking a derivative with respect to $\xi_{I I}$ in eq. (11). We can calculate other physical properties in the same way.

The main point of this investigation is to understand the essential features of the coilglobule transition, specifically, whether the transition is continuous or discontinuous within different models as the concentration of the condensing agent increases. We would like to clarify two things: (i) If a pearl-necklace structure can exit as a thermodynamic state when $N$ goes to infinity, what is the mechanism for that? (ii) What gives a discrete transition? To actually calculate the second generating function in eq. (7), we develop detailed models to calculate $p\left(m_{I}, n_{I}\right)$ and $q\left(m_{I I}, n_{I I}, n_{I I I}\right)$.

Coil. A one-dimensional Ising lattice model is used for the coil part. At high salt concentrations, the repulsive or attractive electrostatic interactions are restricted to neighbouring monomers. One statistical monomer unit is taken to be a section that corresponds to the charge of the condensing agent, commonly +3 or +4 in experiments on DNA [7]. The total number of lattice sites is $n_{I}$. The monomer unit can be occupied by one condensing agent or be unoccupied. The states of the sites are denoted by $j_{1}, j_{2}, \cdots, j_{n_{I}}$. When site $i$ is occupied by a counterion, $j_{i}=1$, while $j_{i}=0$, when it is unoccupied. $w_{00}$ is the energy of interaction between two unoccupied sites, $w_{10}$ and $w_{01}$ are interactions between occupied and unoccupied sites, and $w_{11}$ is the interaction between two occupied sites. The partition function of the Ising lattice is

$$
\Phi\left(\lambda, n_{I}\right)=\sum_{j_{1}, j_{2}, \cdots, j_{n_{I}}=0}^{1} \exp \left[-\frac{w_{j_{1}, j_{2}}+w_{j_{1}, j_{2}}+\cdots-\mu\left(\sum_{i=1}^{n_{I}} j_{i}\right)}{k_{B} T}\right] .
$$

Using the transfer-matrix method, $\Phi\left(\lambda, n_{I}\right)=\chi^{n_{I}}$ is obtained. $\chi$ is the eigenvalue of the transfer matrix. Next, the entropic contribution from the chain conformations is given by $z^{n_{I}}$, which corresponds to a random walk on a lattice with coordination number $z$. In this model, eq. (8) becomes

$$
P(\lambda, \xi)=\sum_{n_{I} \geq 0} \chi^{n_{I}} z^{n_{I}} \xi^{n_{I}} .
$$

Calculating the infinite series with respect to $n_{I}$ gives

$$
P(\lambda, \xi)=\frac{1}{1-\chi z \xi}
$$

where $\chi z \xi<1$ was assumed for convergence and checked in numerical calculations.

Globule. When constructing the free energy of the globule and determining the dependence with respect to the number of particles (monomers and counterions), we note that it is the exponents of these parameters (in a power law) that determine the overall behaviour of the coil-globule transition, while coefficients (numerical constants) have little effect as long as their values are reasonable. It is of particular importance whether the $n / m$ dependence is linear or non-linear. Thus, simple expressions for the free energy will be formulated, starting with a linear contribution and adding non-linear terms. The globule is seen as a spherical object with an ion-crystal-like organisation of the charges, neglecting any conformational entropy of the chain inside the globule. We will take three steps:

(a) First, we consider a cohesive electrostatic energy from ion pairs in the neutral core

$$
E_{l}=-2 l \triangle \epsilon
$$


with $m_{I I}=n_{I I}=l$, where $\Delta \epsilon$ is a cohesive energy per particle. There is only a linear contribution in terms of the number of monomers in the globule part. The degrees of freedom representing the remaining surface charges, $n_{I I I}$, in eq. (9) are neglected here. In this way, we can study the contributions from the combinatorial term, $\Omega_{c-g}$, and from the free energy of the coil part, free of most other effects.

(b) We consider a surface term, originating from a lack of interactions at the surface, which is taken to be spherical. The total interaction energy becomes

$$
E_{l}=-\left(2 l-(2 l)^{2 / 3}\right) \triangle \epsilon
$$

The volume of the sphere is taken to be linear in the total number of particles, $2 l$, in the neutral core, from which it follows that the surface of the neutral core scales as $(2 l)^{2 / 3}$. The degrees of freedom representing surface charges, $n_{I I I}$, in eq. (9) are still neglected here.

(c) Experimentally, it has been reported that a net surface charge remains on the globule part [13]. The resulting electrostatic repulsion between the charges in a thin outer shell is treated as a screened Coulomb interaction, i.e., using the Debye-Hückel approximation. To clearly see the electrostatic effect, we omit the surface term introduced in case (b).

$$
E_{l, n_{I I I}}=-\left(2 l+n_{I I I}\right) \triangle \epsilon+\frac{n_{I I I}^{2}}{(2 l)^{2 / 3}} \frac{l_{B}}{\zeta} \frac{r_{D}}{\zeta}
$$

The last term is the self-energy of the charged thin layer. $l_{B}$ is the Bjerrum length, $r_{D}$ is the Debye screening length, $\zeta$ is a correlation length between ion pairs, and $(2 l)^{2 / 3}$ corresponds to the radius squared. The correlation length inside the ion crystal is considered to be on the same order as the Bjerrum length and the Debye screening length, which gives a trivial constant on the order of one, which we will drop.

The globule part is defined when ten monomer units of the polymer have come together with ten counterions, which sets a lowest cut-off in the sum over states. For case (c), we have,

$$
Q(\lambda, \xi)=\sum_{l \geq 10}^{l_{\max }} \sum_{n_{I I I} \geq 0}^{n_{I I}^{\max }} \exp \left(-\beta E_{l, n_{I I I}}\right) \lambda^{l} \xi^{l} \xi^{n_{I I I}} .
$$

In principle, the summation should go to infinity, but cut-offs, $l_{\max }$ and $n_{I I I}^{\max }$, are introduced for the sake of the numerical calculation, and are set so large that the results are independent of their values. $Q(\lambda, \xi)$ is a polynomial of degree $l_{\max }+n_{I I I}$ with respect to $\xi$.

Substituting eq. (16) into eq. (12), we have,

$$
\eta Q(\lambda, \xi)+\chi z \xi-1=0 .
$$

$\xi_{0}$ is obtained by solving eq. (21) with the appropriate form of $Q(\lambda, \xi)$ (for cases (a) to (c)) for a given $\lambda$. We also use eq. (21) for implicit calculations of partial derivatives such as the one in eq. (13).

Results and Discussion. - The phenomenological input parameters do not affect the qualitative behaviour, therefore, we give them somewhat arbitrary values in units of $k_{B} T$, just to be able to calculate and show the trends numerically. Neighbouring sites where one is occupied by a counterion and the other is unoccupied have net attractions, $w_{10}=w_{01}=$ -3 . Two unoccupied sites have a repulsive electrostatic energy, $w_{00}=1$, due to the bare negative charges of the chain. The interaction between two occupied sites is set to be net attractive, $w_{11}=-1$, to allow for correlations, since an occupied monomer unit contains 

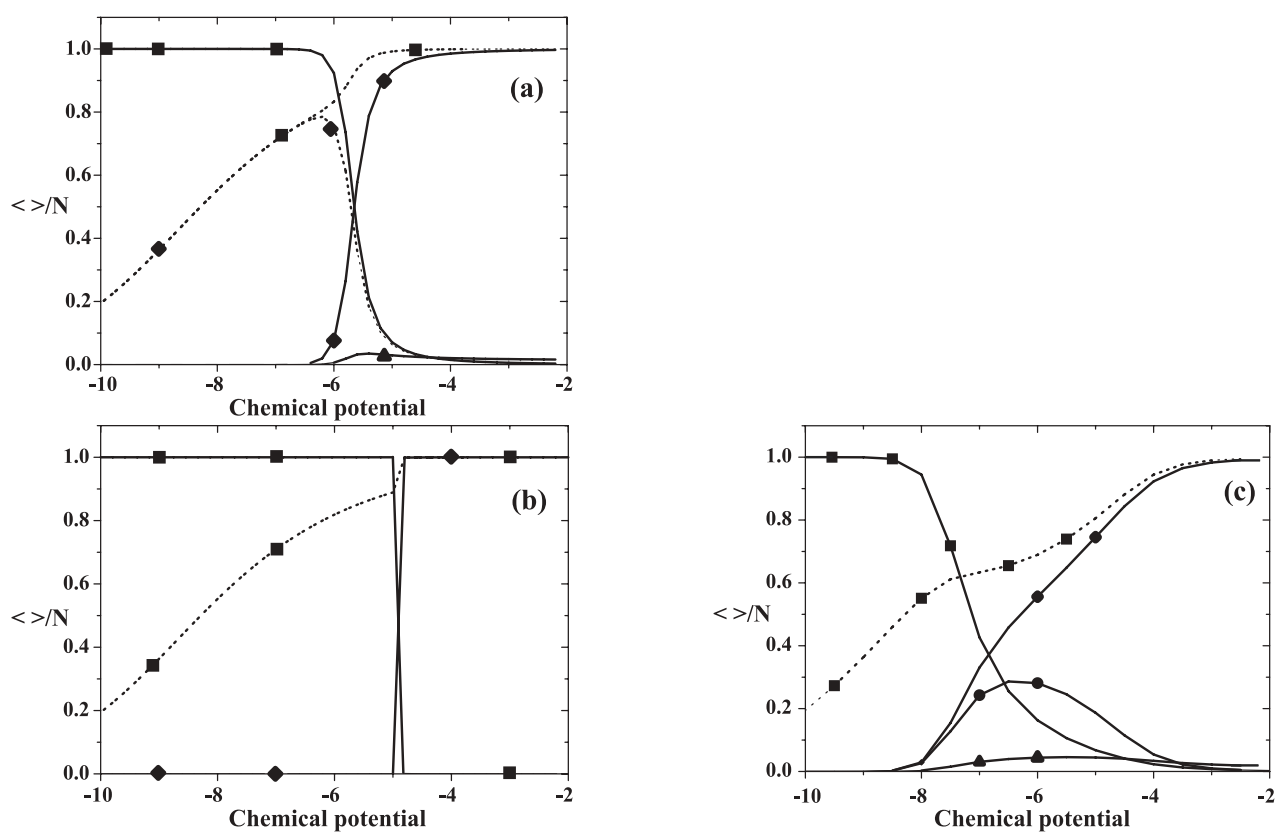

Fig. 2 - Average numbers of monomers, counterions and periodic units divided by the total chain length as functions of the chemical potential of the condensing agent: $n_{I}$ (solid line with squares), $n_{I I}$ (solid line with diamonds), $n_{I I I}$ (solid line with circle), $M$ (dotted line with squares), $m_{I}$ (dotted line with diamonds), and $f$ (solid line with triangles).

one multivalent counterion and several monovalent polymer charges. The strength of the electrostatic interactions inside the globule is represented by $\triangle \epsilon=4$ and for the estimation of the conformational entropy of the chain, the lattice constant is given a value of $z=8$.

Fig. 2a shows case (a). A continuous transition is observed with an interval in which pearlnecklace structures can exist as an equilibrium state. The fraction of partial globules, $\langle f\rangle / N$, has a maximum in the middle of the cross-over region and decreases slowly as the chemical potential of the condensing agent is increased. Note that the reason for the appearance of a partial globule state, i.e., globules connected by coils, is different from the Rayleigh instability, since globules are completely neutralised by counterions in the model. When there is only a linear contribution in the free energy of a globule, it is better to have several local globules along chain to increase the entropy arising from the combinatorial term, $\Omega_{c-g}$, i.e., from the mixing of the coil and globule states. This is analogous to a one-dimensional Ising model, where a mixture of 'spin up' and 'spin down' is favoured for entropic reasons and it follows from having a model that recognises two distinct states locally in the chain.

Fig. 2b shows case (b). The point here is to investigate the effect of a surface term on the transition of the chain. As the concentration of the condensing agent increases, the coil discontinuously collapses into a globule. The surface term in the globule free energy overcomes the combinatorial term $\Omega_{c-g}$, which comes from a one-dimensional representation of the chain, and causes the discrete transition. The origin of this phenomenon is that the chain is a one-dimensional object embedded in a three-dimensional space. To see how the non-linear term works, let us divide eq. (18) by $2 l$ to get the free energy per particle. The linear term gives a constant base line, $-\triangle \epsilon$, and due to the non-linear term, the free energy per particle decreases as $2 l$ increases. This creates cooperativity, i.e., the addition of a particle 
to the globule decreases the free energy per particle.

Fig. 2c shows case (c). A continuous transition with pearl-necklace structures is observed within a wider range than in case (a), although the mechanism is in principle the same. The addition of chain monomers on the surface of the globule is favoured by the cohesive term in eq. (19). Although the repulsive electrostatic interactions disfavour the existence of charges on the surface, there is no cost in the translational entropy of the condensing agent, as there is for monomers in the neutral core, which require an equal number of counterions. Thus, when the concentration of the condensing agent is low, the chain can start to have globules at a lower concentration than in case (a). Towards the end of the transition, the translational entropy costs less, but the system can still profit from having some surface charges.

The fraction of partial globules maintains a significant value in quite a wide range. Consider the separation of one globule with surface charges into two identical globules. The contribution from the cohesive energy does not change, but, due to the electrostatic repulsion in eq. (19), the separation becomes more favourable.

In the normal case, we would expect a combination of case (b) and case (c). With the models and parameter values used here, the effect of the surface term dominates over the electrostatic repulsion when we include both. The coil discontinuously collapses into one globule with the remaining monomer charges on the surface and the charged globule is gradually neutralised by bound counterions as the concentration of the condensing agent increases further. If we tried to balance the surface term and the electrostatic energy with the help of an additional parameter, we could in principle obtain a Rayleigh instability, but separating the charges into smaller globules is not the only mechanism for reducing the electrostatic repulsion. The excess charges can also be neutralised by counterions. Thus, it is important to also consider the translational entropy of the condensing agent. In the experiments, where pearl-necklace structures are observed, we suggest that there is an interplay between all three driving forces.

Y. H. is very grateful to T. Kawakatsu and N. Uchida for the backbone in physics and mathematics, to M. Ullner for discussions and carefully reading of the manuscript, to F. Tanaka for a generating-function method, and to N. Makita and T. Sakaue for discussions.

\section{REFERENCES}

[1] F. J. Solis and M. O. de la Cruz, J. Chem. Phys., 112 (2000) 2030.

[2] P. G. De Gennes, J. Phys. Lett. (France), 36 (1975) 55.

[3] I. M. Lifshitz, A. Y. Grosberg, and A. R. Khokhlov, Rev. Mod. Phys., 50 (1978) 683.

[4] V. V. Vasilevskaya, A. R. Khokhlov, Y. Matsuzawa, and K. Yoshikawa, J. Chem. Phys., 102 (1995) 6595.

[5] G. Swislow, S. T. Sun, I. Nishio, and T. Tanaka, Phys. Rev. Lett., 44 (1980) 796.

[6] C. Wu and S. Zhou, Macromolecules, 28 (1995) 8381.

[7] S. Takagi, K. Tsumoto, and K. Yoshikawa, J. Chem. Phys., 114 (2001) 6942.

[8] M. Ueda And K. Yoshikawa, Phys. Rev Lett., 77 (1996) 2133.

[9] L. J. Kirwan, G. Papastavrou, and M. Borkovec, Nano Lett., 4 (2004) 149.

[10] A. V. Dobrynin, M. Rubinstein, and S. P. Obukhov, Macromolecules, 29 (1996) 2974.

[11] P. Chodanowski and S. Stoll, J. Chem. Phys., 111 (1999) 6069.

[12] F. Oosawa, Polyelectrolytes (Marcel Dekker, New York) 1971

[13] Y. Yamasaki, Y. Teramoto, and K. Yoshikawa, Biophys. J., 80 (2001) 2823. 